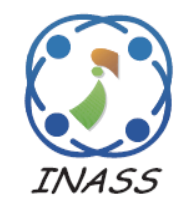

http://www.inass.org/

\title{
Optimization of Shell and Tube Heat Exchanger Design in Organic Rankine Cycle System Using Kinetic Gas Molecule Optimization
}

\author{
Tallapureddy Subba Reddy ${ }^{1 *}$ \\ Thimmasandra Venkataswamy Sreerama Reddy ${ }^{1}$ \\ ${ }^{1}$ Bangalore Institute of Technology, India \\ * Corresponding author's Email: phdsubbareddy@gmail.com
}

\begin{abstract}
Among various types of heat exchanger, Shell and Tube Heat Exchanger (STHX) is highly used in the industrial application. The thermal efficiency of the heat exchanger is considered as a major factor for many applications. A considerable amount of heat is present in the low-temperature geothermal heat source, which is not sufficient to generate the electricity due to its low temperature. Many researches have been made to increase the thermal efficiency of the exchanger by the using of Organic Rankine Cycles (ORC). In this research, the Kinetic Gas Molecule Optimization (KMGO) is used to find the optimized parameter settings for the power plant. The geothermal ORC system is developed in the simulation and other various factors are considered to optimize the performance. The TEMA NFN STHX with a double-segmented baffle is analyzed for single-phase flow model. The different aspects such as baffle cut, baffle leakage, etc., are given as input to the heat exchanger. The proposed technique is evaluated with two different fluids such as R245fa and R134a. The KMGO technique is based on the swarm behavior of gas molecules, which is used to provide the optimized performance. The simulated results are measured and compared it with the existing methods for validation of the performance. The outlet temperature is achieved as $64.52^{\circ} \mathrm{C}$ and the enhancement factor is achieved as 1.88 for the R245fa fluid in STHX. This is achieved due to the KMGO technique identifies the global minima effectively due to the kinetic gas molecules theory, which shows the high efficiency compared to the existing method.
\end{abstract}

Keywords: Double-segmented baffle, Geothermal heat source, Kinetic gas molecule optimization, Organic rankine cycles, Shell and tube heat exchanger, TEMA.

\section{Introduction}

A heat exchanger is a device which is used to recover the thermal energy between fluids, present at different temperature [1]. Heat exchangers are highly used in industrial applications and there are a number of heat exchangers available. The design of heat exchanger consists of many geometric and operational parameters to provide heat transfer while meeting the set of constraints [2]. The STHX is highly used heat exchanger due to its reliability, robustness and versatility. The segmental baffle is a common baffle in the STHX that is highly used in petrochemical industries, waste heat recovery, power generation, air-conditioning and so on [3]. The segmental baffles in heat exchanger provide an improvement in heat transfer of the exchanger [4].
Geothermal power generation showed strong growth in the past decades and the electrical capacity are increased $15.9 \%$ in between the 2010 and 2015 [5]. The global energy crisis and the environmental awareness, create the demand for the renewable energy and these energies are promoted by the developed countries.

The environmental awareness causes to an exploitation of technology of power generation from low-medium temperature heat sources [6]. Much research has been carried out to improve the heat efficiency of the exchanger by using ORC. There are only few studies available for the detailed optimization of the heat exchanger, in terms of efficiency and economic [7]. Artificial Bee Colony (ABC) method has been presented to the optimization of the STHX and their result showed that the ABC provided higher efficiency compared to the 
traditional trial and error method, and conventional Genetic Algorithm [8]. NSGA-II algorithm is applied to the heat recovery in the power system of hybrid PV-diesel [9]. A lot of methods have been followed in the recovery of waste heat or to reduce environmental pollution and energy shortage problem [10]. In this paper, an optimization technique is used to identify the parameters for an effective system of heat transfer. A number of parameters of the heat transfer considered for the KGMO method and outcome of that is analyzed. The fluid used in this method are R245fa and R134a, based on the consideration of the operation conditions cycle and its environmental-friendly characteristics. Different kinds of parameters are given as input to KGMO technique to achieve optimization. The various outcomes have been measured and compared with an existing method for the analysis of efficiency. The simulation result showed that the proposed optimization in STHX has higher performance compared to a state-of-art method.

The paper has been organized as, section 2 contains literature survey, section 3 presents problem definition, Modelling of STHX is in section 4 and Simulation results is provided in section 5 .

\section{Literature survey}

The latest research related to STHX with different aspects are analyzed in this section. Various method in different parameter settings of exchanger were analyzed in this section.

I. Milcheva, F. Heberle, and D. Brüggemann [11] proposed the Taborek version of the Bell-Delaware method for the specific heat exchanger design and the calculation method was applied to the main geometrical parameters like cross flow area, effective number of tubes in the overlapping regions and leakage areas. The simulation was carried out in DYMOLA 2015 FD01 and the achieved outcome was validated by the data of real power plant and the deviation were measured up to $1.14 \%$. The enhancement factor $J_{x}$ was developed and the heat accuracy was attained due to the application to other STHXs with double-segmental baffles. The Taborek version of the Bell-Delaware method was validated by CFD simulated in the ANSYS FLUENT 15.0. The experimental result showed that this method has a higher efficiency in simulation. The function of different fluid is required to test on the simulation for effective performance.

D. Walraven, B. Laenen, and W. D'haeseleer [12] presented a first step toward the optimization of ORCs by optimizing the cycle parameters together with the configuration of STHX. Every heat exchanger has the optimal allocation of a few parameters like heat exchanger surface, pressure drop and pinch-point-temperature difference in the boundary conditions. The different tube configuration was evaluated in this research and concluded that the $30^{\circ}$-tube configuration was used for the single-phase heat exchangers and the $60^{\circ}$-tube configuration was used for the two-phase heat exchangers. The subcritical cycle performance can be increased by adding a second pressure level in the device. More component in the device should be evaluated for better economic optimization.

D. Walraven, B. Laenen, and W. D'haeseleer [13], optimized the plate heat exchanger or STHX together with the cycle configuration. This technique can be applied to the heat exchanger for optimal allocation of some parameters for the given boundary conditions. ORCs with the plate heat exchanger performs better than the ORCs with STHX. The plate heat exchanger has the same geometry on both sides, which can cause the inefficiency. This research showed the influence of the cooling fluid inlet temperature and mass flow on the efficiency of the power plant. This work didn't consider Coolant and additionally effectiveness of the system is needed to measure along with coolant.

F. Calise, C. Capuozzo, A. Carotenuto, and L. Vanoli [14] simulated the model and the model is based on the zero-dimensional energy and mass balances for all the components of the system. This method is also related to the geometrical and design parameters of its components, especially in the case of heat exchanger. This method evaluated the energy and economic performance of STHX in different conditions and design criteria. This allowed to set the geometrical parameters of heat exchanger and evaluated the off-design performance of the system. Hence, this is a useful tool in the preliminary design of the plant. The effectiveness of the ORC is decreased due to increase of the condensing pressure.

S. Cao, J. Xu, Y. Li, and Y. Yan [15] analyzed the condensation of R245fa fluid in a shell-tube condenser. This research suggested that the condenser to operate at weakly inclined flow to reach better thermal performance. Flow patterns and liquid height signals explored the condensation heat transfer mechanisms. The flow is stable in the inclined downflow and unstable for inclined up-flow, which was caused by the vapor-liquid interface wave. Nondimensional parameter analysis identifies the competitions between inertia force and gravity force. The vapor-liquid interface wave is the process to improve the condensation heat transfer for inclined up-flow, and it processed while the decreased liquid 
film thickness on the tube bottom. That also enhances heat transfer for inclined down-flow. A correlation based on the Froude number of vapor phase successfully matched the experimental data. This method is useful for the condenser design and operation of ORC. This method needs to be investigated for the various fluids to analysis the performance.

\section{Problem Statement}

The heat exchanger used in the industries are mainly focused on the maximum efficiency of the heat transfer in long period. There are many problems in using the STHX and some of them are listed below.

- STHX system requires high cost of manufacture and minimizing the manufacturing cost of the heat exchanger with high efficiency helps the manufacturer to cope with the competitor. Most of the previous research mainly focused on the heat efficiency of the system without considering the cost and cost efficiency.

- The coolant temperature in the cooling system is one of the important parameters for analysis. This is well known that the inlet temperature and mass flow have more influence on the performance of STHX.

- The pressure drop gradually increases with increase in cooling water flow rate in heat exchanger. This may increase pumping power, but it decreases the efficiency of the model power plant. However, the rate of increase in pressure drop is very small compared to rate of increase in heat transfer. Hence the rate of increase in pump work has to be reasonably minimal.

To improve the effectiveness of the heat exchanger, the important parameters are needed to consider. The proposed method is used to solve the above-mentioned issues in the heat exchanger.

\section{Modelling of shell and tube heat exchanger}

The ORC preheater of zone model considered in the simulation based on the research [11]. The heat exchanger is divided into several zones and each zone describes the interaction of the cold fluid, the hot fluid, the tubes and the separator. Applying the finite volume method to the developed system from the ordinary differential equations with time derivatives for each zone. This model is the simplification of the distributed models and the parameters change related to the most practical purposes. The partial differential equations used in this work to describe the distributed models. The entire heat exchanger model is implemented based on the methods followed in the research [11]. The exchanger model is designed using the cell models, these are adjusted by changing the parameters like geometry parameters, pressure drop and heat transfer information. These cells suitably modified to process the specific heat exchanger problem. Each cell model represents the control volumes or wall sections and contains the discretized equations for material and energy balance [11].

For considering simulation of the preheater, three cell types are used:

- Refrigerant cells in the shell fluid represent phase change, vapor and liquid phase present,

- Liquid cells from the geothermal fluid led into the tube, which represents the liquid phase present

- Wall cells are used for the thermal conduction of the separator and the tube walls

Shell side design consists of the Heat Transfer Coefficient (HTC) is set as same for the separator wall and at the tube wall. Simulation can support the bidirectional heat flow, i.e., from and to the walls and bidirectional mass flow, i.e., forward and backward. The specific heat exchanger conditions and temperature distributions, respectively determines the actual heat transfer and mass flow direction. Effects of flow regime and heat transfer at the front and at the rear end neglected in the simulation. The pressure drop and the heat transfer area assigned for the each and every refrigerant and liquid cell. In this context, the Gnielinski correlation implemented for the tube-side HTCs. The shell-side HTC for each refrigerant cell (R245fa) measured by using the KMGO algorithm. Pressure drop of cold fluid and hot fluid cells assigned as input parameters from the measured data. This method helps to measure the accuracy of the heat transfer correlation for the shell side.

\subsection{Tube side heat transfer coefficient}

To provide a fully developed turbulent flow occurs in the tube, the Reynolds numbers $R_{e D}$ is set higher than $10^{4}$ for considered load conditions. This flow regime the tube-side heat transfer correlation, Gnielinski is used in the Eq. (1). 


$$
\begin{gathered}
N u_{t}=\left\{\frac{\left(\frac{f}{8}\right) R e_{D} P r}{1+12.7\left(\frac{f}{8}\right)^{\frac{1}{2}}\left(\begin{array}{c}
\frac{2}{3} \\
\operatorname{Pr}-1
\end{array}\right)}\right\}\{1+ \\
\left.\left(\frac{d_{i n}}{L}\right)^{\frac{2}{3}}\right\}\left(\frac{p r_{t}}{p r_{t w}}\right)^{0.11} \times R e_{D} \geq 10^{4}
\end{gathered}
$$

Where Reynolds number is denoted as $R e$ and Prandtl number is denoted as $p r$, diameter is denoted as $D$. The friction factor $\mathrm{f}$ in Eq. (2) is obtained from the correlation of Konakov:

$$
f=\left(1.8 \log _{10} R e_{D}-1.5\right)^{-2} 10^{4} \leq R e_{D} \leq 10^{6}
$$

\subsection{Shell side heat transfer coefficient}

For calculating the average shell-side $\mathrm{HTC} h_{s}$, KGMO optimization technique is used. Which is shown in the Eq. (3).

$$
h_{s}=J_{c} J_{l} J_{r} J_{b} J_{s} h_{i}
$$

\subsubsection{Kinetic gas molecule optimization}

Kinetic Gas Molecule Optimization (KGMO) is a swarm-based algorithm for solving nonlinear problems, which works based on gas molecule theory [17]. The gas molecules are the agents in the search space. Kinetic energy is used for performance measurement and control [18, 19]. Considering a system with agents (gas molecules), the position of the $i^{\text {th }}$ agent is defined in the Eq. (4).

$$
X_{i}=\left(x_{i}^{1}, \ldots, x_{i}^{d}, \ldots v_{i}^{n}\right) \text { for } i=1,2, \ldots, N
$$

Where $v_{i}^{d}$ represents the velocity of the $i^{t h}$ agent in the $d^{\text {th }}$ dimension and the velocity of the $i^{\text {th }}$ agent is presented in the Eq. (5).

$$
V_{i}=\left(v_{i}^{1}, \ldots v_{i}^{d}, \ldots, v_{i}^{n}\right), \text { for }(i=1,2, \ldots, N)
$$

Where $v_{i}^{d}$ represents the velocity of the $i^{\text {th }}$ agent in the $d^{t h}$ dimension. Fundamental equations in KMGO are as below. The kinetic energy, which is defined in the Eq. (6).

$$
\begin{aligned}
& \quad k_{i}^{d}(t)=\frac{3}{2} N b T_{i}^{d}(t), K_{i}\left(k_{i}^{1}, \ldots k_{i}^{d}, \ldots, k_{i}^{n}\right), \text { for } \\
& (i=1,2, \ldots, N)
\end{aligned}
$$

Where $N$ is the number of gas molecules, $b$ is the Boltzmann constant and $T_{i}^{d}(t)$ is the temperature of $i^{\text {th }}$ agent in the $d^{\text {th }}$ dimension at time $t$. The velocity of the molecule is updated by:

$$
\begin{array}{r}
v_{i}^{d}(t+1)=T_{i}^{d}(t) w v_{i}^{d}(t)+ \\
C_{1} \operatorname{rand}_{i}(t)\left(\operatorname{gbest}^{d}-x_{i}^{d}(t)\right)+ \\
C_{2} \operatorname{rand}_{i}(t)\left(\text { pbest }_{i}^{d}(t)-x_{i}^{d}(t)\right)
\end{array}
$$

Where $T_{i}^{d}(t)$ for converging molecules reduces exponentially over time, calculated in the Eq. (8).

$$
T_{i}^{d}(t)=0.95 \times T_{i}^{d}(t-1)
$$

The vector pbest $_{i}=$ $\left(\right.$ pbest $_{i}^{1}$, pbest $_{i}^{2}, \ldots$, pbest $\left._{i}^{n}\right)$ provides the best previous position of the $i^{t h}$ gas molecule and the gbest $=\left(\right.$ gbest $^{1}$, gbest $^{2}, \ldots$, gbest $\left.^{n}\right)$ is the best previous position among all the molecules in the container. The random particles are applied to initialize the velocity and the position of each particle, which are present in the corresponding ranges. $\left[-v_{\text {min }}, v_{\text {max }}\right]$ is the limits of the gas molecules velocity. If $\left|v_{i}\right|>v_{\max }$, then $\left|v_{i}\right|=v_{\max } . w$ is the inertia weight that shows the gas molecules resistance to slow its movement. The $\operatorname{rand}_{i}(t)$ is a uniform random variable in the interval $[0,1]$ at time $t$, used to provide a randomized characteristic to the search algorithm, where $C_{1}, C_{2}$ are acceleration constants. The mass $m$ of each gas molecule is a random number is in the range of $0<m \leq 1$, which remains constant throughout the execution of the algorithm as the container is consider to have only one type of gas at any one time. The random number is used to simulate different types of gases in different execution of the algorithm.

According to [18], the position is updated for each unit of time interval by:

$$
x_{t+1}^{i}=\frac{\sqrt{2\left(\Delta k_{i}^{d}\right)}}{m}(t+1)+v_{i}^{d}(t+1) x_{i}^{d}(t)
$$

The minimum fitness function is found using:

$$
\begin{aligned}
& \text { pbest }_{i}=f\left(x_{i}\right) \text { if } f\left(x_{i}\right)<f\left(\text { pbest }_{i}\right) \\
& \text { gbest }_{i}=f\left(x_{i}\right) \text { if } f\left(x_{i}\right)<f\left(\text { gbest }_{i}\right)
\end{aligned}
$$

Each gas molecule tries to change its position $\left(x_{i}^{d}\right)$ using the distance between the current position and pbest $_{i}^{d}$, and the distance between the current position and $g b e s t_{i}$. This method considers different effects to reduce the ideal HTC $h_{i}$ on a tube bank in cross flow. A deviation from the ideal cross flow 
Table 1. Correction factors considered in KMGO

\begin{tabular}{|c|c|}
\hline Correction factor (CF) & Influences/effects taken into account \\
\hline$J_{c}:$ baffle cut CF & Effect of window respectively of baffle cut \\
\hline$J_{l}:$ baffle leakage CF & Effect of leakages between shell and baffles and between tubes and baffles \\
\hline$J_{r}:$ laminar flow CF & $\begin{array}{c}\text { Effect of flow between inner shell wall and the tube bundle and the influence of pass } \\
\text { partition lanes }\end{array}$ \\
\hline$J_{b}$ & $\begin{array}{c}\text { Effect of the flow between inner shell wall and the tube bundle and the influence of pass } \\
\text { partition lanes }\end{array}$ \\
\hline $\begin{array}{c}J_{s}: \text { unequal baffle } \\
\text { spacing CF }\end{array}$ & Effect of unequal inlet or outlet baffle spacing different from central baffle spacing \\
\hline
\end{tabular}

Table 2. Heat transfer coefficient and Reynolds's number of shell and tube heat exchanger

\begin{tabular}{|c|c|c|c|c|c|c|c|c|c|}
\hline & $\boldsymbol{\alpha}_{\boldsymbol{s}, \boldsymbol{m i n}}$ & $\boldsymbol{\alpha}_{\boldsymbol{s}, \boldsymbol{m a x}}$ & $\boldsymbol{R e}_{\boldsymbol{s , \boldsymbol { m i n }}}$ & $\boldsymbol{R e}_{\boldsymbol{s , \boldsymbol { m a x }}}$ & $\boldsymbol{\alpha}_{\boldsymbol{t}, \boldsymbol{m i n}}$ & $\boldsymbol{\alpha}_{\boldsymbol{t s}, \boldsymbol{m a x}}$ & $\boldsymbol{R e}_{\boldsymbol{t}, \boldsymbol{m i n}}$ & $\boldsymbol{R e}_{\boldsymbol{t}, \boldsymbol{m a x}}$ & $\frac{\boldsymbol{\alpha}_{\boldsymbol{t}, \boldsymbol{m a x}}}{\boldsymbol{\alpha}_{\boldsymbol{s}, \boldsymbol{m a x}}}$ \\
\hline Unit & $(\mathrm{W} / \mathrm{m} 2$ & $(\mathrm{W} / \mathrm{m} 2$ & - & - & $(\mathrm{W} / \mathrm{m} 2$ & $(\mathrm{W} / \mathrm{m} 2$ & - & - & - \\
\hline Design & 1204 & $\mathrm{~K})$ & - & - & $\mathrm{K})$ & $\mathrm{K})$ & - & - & - \\
\hline
\end{tabular}

regime is consider in form of several correction factors $\left(J_{c}, J_{l}, J_{r}, J_{b}, J_{s}\right)$. In Table 1 the corresponding effects and correction factors are listed. The ideal HTC $h_{i}$ is determined by the HTC for flow across the ideal tube bank [20].

$$
h_{i}=\left\{\frac{\left(j_{i} C_{p s} G_{s}\left(\Phi_{s}\right)\right)^{n}}{P r_{s}^{\frac{2}{3}}}\right\}
$$

Where $C_{p s}$ is the shell side specific Heat capacity, $G_{s}$ is the shell side mass flow rate and $\Phi_{s}$ is Shell side viscosity correlation factor.

\section{Design and simulation result}

The STHX was simulated in the MATLAB with the system specification of 8GB RAM, and Intel i3 processor. The Heat Transfer Coefficient and Reynolds's number of the STHX is shown in Table 2. For the considered load conditions, the tubes Reynolds number $R_{e D}$ is higher than $10^{4}$, i.e. a fully turbulent flow occurs. The Heat transfer coefficient is denoted as $\alpha$ and Reynolds number as $R e$, and subscripts like $s$ denotes shell, ss denotes shell side, min and max denotes minimum and maximum, and time is denoted as $t$.

STHX is simulated in the MATLAB software and optimized parameter for effective performance. The cell model implemented and performed optimization technique in the developed model of STHX. The geothermal ORC power plant [21] was used to analyze the performance. The data presented in the research doesn't reflect the maximum performance that can be obtained by the ORC plant. As a number of adverse factors like heat exchangers fouling,

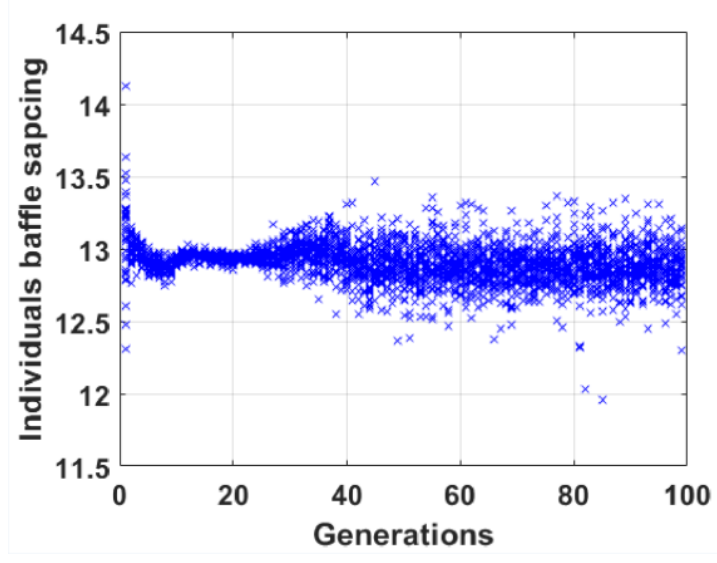

Figure.1 Individual baffle spacing for various generations

presence of non-condensable gases, etc., present in the power plant may cause to decrease the performance, which are not considered in the research. A detailed description and thermodynamic analysis of the entire geothermal power plant is provided in the research [21].

\subsection{Design of shell and tube heat exchanger}

The shell side cross flow area and the effective tube row number are used as input parameter for calculating baffle leakage $C F\left(J_{l}\right)$ and laminar flow $C F\left(J_{r}\right)$. The wing baffles and central baffles are in build-in in the simulation. The wing baffles are symmetric and have equal dimension at the same baffle section. The central baffle in the STHX will vary based on the size, window tube number and form.

\subsection{Shell tube cross flow area}

The symmetry settings are used and assumed that the flow in the window right and window left are 
same. Then, the shell side cross flow area $S_{m, l}$ and $S_{m, r}$ are equal. So Shell side cross flow area is calculated using the Eq. (13) and $N_{t c f}$ denotes tube number in one row that is same as in overlapping region.

$$
S_{m}=S_{m, l}+S_{m, r}=L_{b c}\left(b_{x}-N_{t c f} d\right) \cdot 2
$$

\subsection{Effective tube row number}

The effective number of rows in overlapping regions is $N_{t c c, l}$ and $N_{t c c, r}$ are added to measure tube row number in cross flow $N_{t c c}$, as shown in Eq. (14).

$$
N_{t c c}=N_{t c c, l}+N_{t c c, r}
$$

To calculate the wing-window tube number $N_{t w, w}$, the left and right wing windows $N_{t w, l}$ and $N_{t w, r}$ are added, given in Eq. (15).

$$
N_{t w, l}=N_{t w, l}+N_{t w, r}
$$

\subsection{Wing baffle and central baffle}

The wing baffle and central baffle have different settings like number of tubes in the baffle window. The average window tube number in the left and in the right wing windows are used. Then, the baffle tube fraction $F_{w}$ is measured by dividing the average window tube number by the total tube Number $N_{t}$ in the Eqs. (16) and (17).

$$
\begin{aligned}
& N_{t w}^{\text {aver }}=0.5\left(N_{t w, w}+N_{t w, C}\right) \\
& F_{w}=\frac{N_{t w}^{\text {aver }}}{N_{t}}
\end{aligned}
$$

The same is followed to the shell-to-baffle and tube-to-baffle leakage area.

\section{Simulation results}

Several parameters selected using the KMGO technique and the optimized values are chosen for efficient performance. The individual baffle spacing is chosen for the various generations in KMGO method. The individual baffle spacing is selected in various generations represented in the Fig. 1. The proposed method is evaluated on the different aspects like enhancement factor, Geothermal water outlet temperature, etc. The proposed method evaluated in the same environment followed in the research [11] and results is compared with Bell-Delaware method. The bell-Delaware method was used in the research [11] and the proposed technique is compared with the simulated results from [11].

Taborek version of the Bell-Delaware method for the specific heat exchanger design and the calculation method was applied to the main geometrical parameters like cross flow area, effective number of tubes in the overlapping regions and leakage areas [11]. This research considered the preheaters of an ORC system from the TEMA NFN type with double segmental baffles and this configuration is a two pass shell with a longitudinal baffle in the middle is called separator. The fluid R245fa is used into the shell and warmed up to the boiling point. The hot medium is the geothermal fluid that led into the tubes. The Taborek version of the Bell-Delaware method provide the considerable performance using the geometrical aspects like effective number of tubes in the overlapping region, leakage area, and cross flow area to specific heat exchanger design by adjusting calculation method.

Table 3. Simulation result for the LT preheater using R245fa fluid

\begin{tabular}{|c|c|c|c|}
\hline Operating point & Unit & $\begin{array}{c}\text { Bell- } \\
\text { Delaware } \\
\text { method [11] }\end{array}$ & $\begin{array}{c}\text { KGMO } \\
\text { technique }\end{array}$ \\
\hline Enhancement factor $J_{x}$ & - & 1.51 & 1.8864 \\
\hline R245fa inlet temperature & ${ }^{\circ} \mathrm{C}$ & 19.9 & 19.9000 \\
\hline R245fa outlet temperature & ${ }^{\circ} \mathrm{C}$ & 59.34 & 64.5239 \\
\hline R245fa inlet pressure & bar & 5.48 & 5.4800 \\
\hline R245fa outlet pressure & bar & 5.18 & 4.3800 \\
\hline R245fa mass flow & $\mathrm{kg} / \mathrm{s}$ & 85.55 & 88.3333 \\
\hline Geothermal water inlet temperature & ${ }^{\circ} \mathrm{C}$ & 66.64 & 66.6400 \\
\hline Geothermal water outlet temperature & ${ }^{\circ} \mathrm{C}$ & 47.77 & 52.1439 \\
\hline Geothermal water inlet pressure & $\mathrm{bar}$ & 15 & 15 \\
\hline Geothermal water outlet pressure & $\mathrm{bar}$ & 15 & 4.3800 \\
\hline Thermal water mass flow & $\mathrm{kg} / \mathrm{s}$ & 58.1 & 61.1839 \\
\hline Relative deviation R245fa outlet temperature & $\%$ & 0.38 & 0.3214 \\
\hline Relative deviation geothermal fluid outlet temperature & $\%$ & -0.19 & -0.1400 \\
\hline
\end{tabular}


Table 4. Simulation result for the LT preheater using R134a fluid

\begin{tabular}{|c|c|c|}
\hline Operating point & Unit & KGMO technique \\
\hline Enhancement factor $J_{x}$ & - & 1.2329 \\
\hline R134a inlet temperature & ${ }^{\circ} \mathrm{C}$ & 19.9000 \\
\hline R134a outlet temperature & ${ }^{\circ} \mathrm{C}$ & 42.1741 \\
\hline R134a inlet pressure & bar & 5.4800 \\
\hline R134a outlet pressure & bar & 6.5800 \\
\hline R134a mass flow & $\mathrm{kg} / \mathrm{s}$ & 67.0000 \\
\hline Geothermal water inlet temperature & ${ }^{\circ} \mathrm{C}$ & 66.6400 \\
\hline Geothermal water outlet temperature & ${ }^{\circ} \mathrm{C}$ & 27.5941 \\
\hline Geothermal water inlet pressure & $\mathrm{bar}$ & 15.0000 \\
\hline Geothermal water outlet pressure & $\mathrm{bar}$ & 17.3000 \\
\hline Thermal water mass flow & $\mathrm{kg} / \mathrm{s}$ & 38.3141 \\
\hline Relative deviation R134a outlet temperature & $\%$ & 0.4032 \\
\hline Relative deviation geothermal fluid outlet temperature & $\%$ & -0.2600 \\
\hline
\end{tabular}

Table 3 presents the simulated results. The proposed method is also evaluated based on the fluid R134a and results are shown in Table 4.

The simulated result is provided in the Table $3, J_{x}$ is developed using linearly on the shell fluid mass flow rate $M_{S}$ as follows

$$
J_{x}=0.0058 . M_{s}+1.0102
$$

In this research, the LT preheater of the ORC is considered for simulation and compared it with BellDelaware method followed by research [16]. The different aspect was chosen for the simulation, as shown in Table 3, and correlation for the enhancement factor $J_{x}$ is chosen.

Simulation results having the little deviation in the range of $0.52 \%$ for the shell fluid and lower than $0.26 \%$ for the tube side. Temperature of the geothermal fluid inlet is also smaller and the rate of mass flow is 88.33 . Hence, this method can be used to optimize the performance of the heat exchanger. This optimization technique has been analyzed in the fluid of R134a and results are shown in Table 4. The simulated result of the R134a is low compared to the $\mathrm{R} 245 \mathrm{fa}$ in terms of heat transfer. The mass flow is provided as $67 \mathrm{~kg} / \mathrm{s}$ and the $J_{x}$ is calculated as 1.2329 .

The KMGO technique identifies the global minima effectively due to the kinetic gas molecules theory, which shows the high efficiency compared to the existing method.

\section{Conclusion}

The STHX is simulated based on the TEMA NFN with double segmental baffles. The optimization technique is applied to the heat exchanger to attain the design parameters for effective performance. The tube side heat transfer coefficient is calculated and in the shell side, KMGO optimization technique is applied. The parameters considered in the optimization are HTC $h_{i}$ and correction factors $J_{c}, J_{l}, J_{r}, J_{b}$ and $J_{s}$. Using the optimization of KMGO technique, the outlet temperature increased. The correlation factor $J_{x}$ is measured and this represents the effect of increased turbulence. The KMGO technique increases the performance of the heat exchanger. The proposed technique is evaluated on the two types of fluid such as R245fa and R134a. Higher performance achieved in the fluid of R245fa with the outlet temperature value of $64.52^{\circ} \mathrm{C}$ and enhancement factor of 1.88. In the future work, the different parameter settings can be measured for increasing efficiency of the heat exchanger.

\section{References}

[1] R.V. Rao and A. Saroj, "Economic optimization of shell-and-tube heat exchanger using Jaya algorithm with maintenance consideration", Applied thermal Engineering, Vol.1, No.16, pp.473-487, 2017.

[2] R.V. Rao and A. Saroj, "Constrained economic optimization of shell-and-tube heat exchangers using elitist-Jaya algorithm", Energy, Vol.128, pp.785-800, 2017.

[3] Y. Lei, Y. Li, S. Jing, C. Song, Y. Lyu, and F. Wang, "Design and performance analysis of the novel shell-and-tube heat exchangers with louver baffles", Applied Thermal Engineering, Vol.125, pp.870-879, 2017.

[4] X. Gu, M. Wang, Y. Liu, and S. Wang, "Multiparameter optimization of shell-and-tube heat exchanger with helical baffles based on entransy theory", Applied Thermal Engineering, Vol.130, pp.804-813, 2018.

[5] F. Heberle, C. Schifflechner, and D. Brüggemann, "Life cycle assessment of Organic Rankine Cycles for geothermal power generation considering low-GWP working fluids", Geothermics, Vol.64, pp.392-400, 2016. 
[6] F. Calise, C. Capuozzo, A. Carotenuto, and L. Vanoli, "Thermoeconomic analysis and offdesign performance of an organic Rankine cycle powered by medium-temperature heat sources", Solar Energy, Vol.103, pp.595-609, 2014.

[7] D. Walraven, B. Laenen, and W. D'haeseleer, "Economic system optimization of air-cooled organic Rankine cycles powered by lowtemperature geothermal heat sources”, Energy, Vol.80, pp.104-113, 2015.

[8] [8] M. Mirzaei, H. Hajabdollahi, and H. Fadakar, "Multi-objective optimization of shell-and-tube heat exchanger by constructal theory", Applied Thermal Engineering, Vol.125, pp.9-19, 2017.

[9] [9] B. D. Raja, R. L. Jhala, and V. Patel, "Manyobjective optimization of shell and tube heat exchanger", Thermal Science and Engineering Progress, Vol.2, pp.87-101, 2017.

[10] C. Zhang, C. Liu, S. Wang, X. Xu, and Q. Li, "Thermo-economic comparison of subcritical organic Rankine cycle based on different heat exchanger configurations", Energy, Vol.123, pp.728-741, 2017.

[11] I. Milcheva, F. Heberle, and D. Brüggemann, "Modeling and simulation of a shell-and-tube heat exchange for Organic Rankine Cycle systems with double-segmental baffles by adapting the Bell-Delaware method", Applied Thermal Engineering, Vol.126, pp.507-517, 2017.

[12] D. Walraven, B. Laenen, and W. D'haeseleer, "Optimum configuration of shell-and-tube heat exchangers for the use in low-temperature organic Rankine cycles", Energy Conversion and Management, Vol.83, pp.177-187, 2014.

[13] D. Walraven, B. Laenen, and W. D'haeseleer, "Comparison of shell-and-tube with plate heat exchangers for the use in low-temperature organic Rankine cycles", Energy Conversion and Management, Vol.87, pp.227-237, 2014.

[14] F. Calise, C. Capuozzo, A. Carotenuto, and L. Vanoli, "Thermoeconomic analysis and offdesign performance of an organic Rankine cycle powered by medium-temperature heat sources", Solar Energy, Vol.103, pp.595-609, 2014.

[15] S. Cao, J. Xu, Y. Li, and Y. Yan, "Condensation heat transfer of R245fa in a shell-tube heat exchanger at slightly inclined angles", International Journal of Thermal Sciences, Vol.115, pp.197-209, 2017.

[16] M. Thorade and A. Saadat, "Partial derivatives of thermodynamic state properties for dynamic simulation", Environmental

Earth

Sciences, Vol.70, No.8, pp.3497-3503, 2013.

[17] S. Moein, R. Logeswaran, and M. Faizal bin Ahmad Fauzi, "Detection of heart disorders using an advanced intelligent swarm algorithm", Intelligent Automation \& Soft Computing, Vol. 23, No.3, pp.419-424, 2017.

[18] S. Moein and R. Logeswaran, "KGMO: A swarm optimization algorithm based on the kinetic energy of gas molecules", Information Sciences, Vol.275, pp.127-144, 2014.

[19] M. Basu, "Kinetic gas molecule optimization for nonconvex economic dispatch problem", International Journal of Electrical Power \& Energy Systems, Vol.80, pp.325-332, 2016.

[20] M. Fesanghary, E. Damangir, and I. Soleimani, "Design optimization of shell and tube heat exchangers using global sensitivity analysis and harmony search algorithm", Applied Thermal Engineering, Vol.29, No.5-6, pp.1026-1031, 2009.

[21] F. Heberle, T. Jahrfeld, and D. Brüggemann, "Thermodynamic analysis of double-stage organic rankine cycles for low-enthalpy sources based on a case study for 5.5 mwe power plant kirchstockach (germany)", In: Proc. of the World Geothermal Congress, pp.19-25, 2015. 